Article

\title{
Perceptions of school management on the relationship between school nutrition and development of non-communicable dis- eases in a rural South African district: A qualitative study
}

\author{
Sibusiso C. Nomatshila ${ }^{1,}$, Teke R. Apalata ${ }^{2}$ and Sikhumbuzo A. Mabunda ${ }^{3}$ \\ 1 Department of Public Health, Faculty of Health Sciences, Walter Sisulu University, Mthatha 5117, South \\ Africa. E-mail: snomatshila@wsu.ac.za Phone: 087351 3548, Mobile: 0833751115 \\ 2 Department of Laboratory Medicine, Faculty of Health Sciences, Walter Sisulu University, Mthatha 5117, \\ South Africa. E-mail: tapalata@wsu.ac.za \\ 3 The George Institute for Global Health, University of New South Wales, Sydney, Australia. Email: sma- \\ bunda@georgeinstitute.org.au \\ * Correspondence: esibusiso.nomatshila@gmail.com; Tel.: +2783 3751115
}

\begin{abstract}
Globally, noncommunicable diseases (NCDs) were responsible for 41 million deaths in 2016, with the majority of these occurring in low and middle-income countries. These diseases were on the rise as a result of unhealthy, low-quality, and unbalanced diets, which resulted in overweight and obesity. The National School Nutrition Programme (NSNP) was created to regulate the foods sold to schoolchildren. To ascertain school management teams' perspectives on the relationship between the NSNP and the development of lifestyle diseases. A phenomenological qualitative study using Focus Group Discussions among 16 purposively selected members of the School Management Teams were conducted in Mt Frere, Eastern Cape in 2016. The narrative data was analyzed using Tesch's eight-phase thematic analysis approach. The data analysis revealed two themes (NSNP and the vendor system) and six sub-themes. The NSNP was viewed as making a significant contribution to children's food security, thereby improving academic output. However, reengineering of the NSNP was need through improved budgeting and inclusion of breakfast in the menu to control NCDs risk factors. The current implementation of the vendor system did not support reduction of NCDs risk factors. Improved implementation of the guide to vendor system is needed.
\end{abstract}

Keywords: Nutrition program; vendor system; qualitative study; obesity; non-communicable diseases

\section{Introduction}

In 2018, the World Health Organization (WHO) reported that non-communicable diseases (NCDs) accounted for around 41 million (or 71\%) of all worldwide deaths, of which $77 \%$ came from low- and middle income-countries (LMICs) [1]. About $85 \%$ of these deaths were premature deaths [1]. This represented a $3 \%$ increase over four years from 2014 , with NCDs accounting for 38 million of all global deaths, with most $(68 \%)$ of these deaths occurring in LMICs [2,3]. Non-communicable diseases like cardiovascular diseases, diabetes, some respiratory diseases and cancers are reported to have the potential of increasing exponentially by the year 2040 especially in LMICs and will account for many premature deaths [4].

Non-communicable diseases are on the rise because of a variety of factors, including unhealthy, low-quality diets; excessive and unbalanced intake leads to overweight and obesity, both of which are risk factors for NCDs [5]. Diet-related NCDs impose a significant burden on populations, families, and the health-care system [6]. Unhealthy diets, poor quality diets and malnutrition are reported as the major risk factor for NCDs, and have been attributed to dramatic global changes in food production, marketing, supply and consumption [5] 
The National School Nutrition Programme (NSNP) was developed to assist in providing proper nutrition and improving the children's wellbeing through guidance on the structure of the lunch box, foods to be served at schools, guidelines on the establishment of tuck-shops at school, what these tuck-shops should sell to children and teachers, and the establishment of school gardens [7]. In a study conducted in 2015 by Gresse and colleagues in South Africa, it transpired that there was a challenge with the management of tuck-shops, arising from the fact that they did not form part of the school system and were not accountable to the same structures as schools [8]. As such, adequate training for their managers is of paramount importance.

The School Management Teams (SMTs) are empowered by the South African Schools Act (SASA) (1996) with the responsibility of governance to schools whilst they oversee the implementation of a successful NSNP through promotion of the availability of healthy food alternatives from school tuck shops/vendors in school premises [7,9]. Challenges in the management of tuck-shops and the general vendor system in schools as part of the NSNP exposes school children to unregulated sale of low cost and prohibited food items with no nutritional advantage [10] thus weakening controls of NCDs risk factors. These weakened controls are exacerbated by the flaws in the functioning of the NSNP: inconsistent food distribution, decayed and poor quality food, limited variation, with no fruit and vegetables [11,12]. This situation defeats the purpose of the establishment of the NSNP, resulting in children having to carry money to school in order to obtain alternate meals or snacks that are often high in fat content and calories as part of their daily diets [13]. A study conducted in Nepal revealed poor coordination among relevant stakeholders was identified as a barrier to the success of school nutrition programs [14]. Shrestha et al. proposed better coordination and collaboration to continue efforts to implement and strengthen the program. The purpose of this study was to ascertain school management teams' perceptions of the relationship between the school nutrition program and the development of NCDs.

\section{Materials and Methods}

\subsection{Study design}

This phenomenological qualitative study using Focus Group Discussions to understand the perceptions of school governance leadership about the implementation of the National School Nutrition Programme, and its effects on the development of the NCDs.

\subsection{Setting}

This study was conducted in Mt Frere, a rural town in the north-western part of the Alfred Nzo District Municipality. Most of the population resides in the rural areas surrounding the town. Alfred Nzo municipality had a population of 199620 in 2016 of which more than $42 \%$ had not completed their secondary education [15].

\subsection{Population}

Members of school management teams (two principals, one deputy principal, three department heads, eight parent representatives, and two educators) took part in the study. These participants were seen as having positions of authority in schools and were thought to be critical for the Focus Group Discussions (FGDs). Each focus group had 6-10 participants. Even though these participants did not represent the general population of school management teams in schools throughout the Eastern Cape, they did represent school management teams in Mt Frere.

\subsection{Sampling procedure}

Purposive sampling was used to select participants for the FGDs on selected days for data collection. Each Focus Group Discussion had 6-10 participants comprising of all members of the school management to explore their perceptions on the associations of the School Nutrition Programme and the development of NCDs in Mt Frere. Mt Frere 
education district has 217 schools. However, the population of interest was in 25 schools of which $5(20 \%)$ schools were sampled through simple random sampling resulting in one secondary and four primary schools. School management included educator and parent representatives.

\subsection{Data collection}

\subsubsection{Focus group discussions}

All participants were given the opportunity to take part in focus group discussions (FGDs) in exploring the perceptions of school administration's on NCDs and what could be done to prevent them. Two focus group discussions (FGDs) were held, each lasting minimum of 60 minutes. An expert in the field of public health and health promotion moderated the discussion. An interview guide was used to ask open-ended questions. All focus group discussions were held in IsiXhosa, a widely spoken local language in the area. Discussions were audio-recorded and then meticulously transcribed for analysis by a skilled transcriber.

\subsection{Data analyses}

Descriptive data from the FGDs were analyzed immediately after FGDs using the Tesch's eight phases thematic analysis approach [16]. Permission was sought from the participants to voice-record the discussions of FGDs. All data collected were precisely transcribed from the sound recorder to avoid misinterpretation of useful information. The transcriptions were done from vernacular (isiXhosa) into English and back to IsiXhosa to avoid distortion. The audio recorder was run a number of times while being stopped intermittently to accurately capture substance in the recordings. The field notes were also typed, and their contents infused into the main transcripts for data comprehensiveness. The final transcripts were reviewed by the transcriber and other team members to get clarity and consistency of the information to real meaning conveyed. Themes and subthemes were developed from categories with similar meanings; an independent coder was used to confirm the themes. A test-retest coding and clustering approach was used. All sound recordings were, thereafter, destroyed to maintain confidentiality principles.

\subsection{Ethics and consent}

The study was approved by the Walter Sisulu University's Human Research Ethics and Biosafety Committee for ethical approval (070/15). Permission to conduct the study in schools was also sought from the Eastern Cape province's Departments of Health and Basic Education. Participants provided written informed consent with full details about the study and reporting channels. All research records were kept in a locked filing system, and all electronic data was coded and secured with a password-protected file. Participants' identities were not recorded or disclosed in any way.

\subsection{Reliability and validity}

To check reliability, relevance of research questions, phrasing, questions format and ability to measure what was intended, the instrument for data collection was based on questions validated through a small-scale pilot of 5 (five) people not participating in the core study. Test-and-re-test approach was used to confirm accuracy of the adjusted questions after the pilot study.

\subsection{Trustworthiness}

This relates to the trustworthiness of the qualitative research study. Credibility, confirmability, transferability and dependability in the study were implemented in line with [17]. This was accomplished by increasing the amount of time spent with participants to ensure consistency and a better understanding of their responses to NCD risk factors. Respondents were encouraged to take ownership of the research as a means of improving their knowledge and skills in preventing NCD risk factors. The use of tape recording and 
note taking ensured that a wide range of perspectives and information were collected and aligned to ensure accuracy in recording responses.

\section{Results}

The data for this study was collected from two FGDs with a total of 16 participants from school management teams in Mt Frere, Eastern Cape province. There were 10 participants in the first FGD which lasted for 60 minutes whilst the second FGD had six participants and took 70 minutes to complete.

The data resulted in two themes and six sub-themes as presented below:

\begin{tabular}{|c|c|c|}
\hline & Theme & Subthemes \\
\hline \multirow{4}{*}{1.} & \multirow{4}{*}{$\begin{array}{l}\text { National School Nutrition Pro- } \\
\text { gramme }\end{array}$} & $1.1 \quad$ Poverty \\
\hline & & School attendance \\
\hline & & 1.3 Menu budgeting \\
\hline & & Food handling and preparations \\
\hline & \multirow{2}{*}{ Vendors system } & $2.1 \quad$ Sold items \\
\hline & & $\begin{array}{ll}2.2 & \text { District support }\end{array}$ \\
\hline
\end{tabular}

\subsection{THEME 1: NATIONAL SCHOOL NUTRITION PROGRAMME (NSNP)}

\subsubsection{Subtheme 1: Poverty}

The implementation of the NSNP, policy framework and implementation, support system and compliance were expressed as key areas in the school system. Participants expressed their appreciation of the NSNP and indicated that it was a good initiative that contributed meaningfully towards alleviation of poverty and in improving academic performance among learners.

"It is good for the case of children in our areas because government came and looked at quintiles which have parents who are not working and under poverty".

Participant 1, FGD 1

The introduction of the NSNP was commended in that it provided a long-needed approach to enhancing school performance.

\subsubsection{Subtheme 2: School attendance}

"It is good because children know that they surely will have something to eat at school and that improves school attendance".

\section{Participant 1, FGD 2}

Educators felt that the introduction of the NSNP with its standard rule of serving the meals at least before $10 \mathrm{~h} 00$ in the morning provided a chance of mediating risks of skipping breakfast for children from these communities in Mt Frere. There was a feeling that if school managements were going to be given an opportunity to apply their minds as schools and ensured the implementation of the policy in way that suited their local situations, more could be done in developing diets and meals system that could generate better health outcomes for their children.

\subsubsection{Subtheme 3: Menu budgeting}

One of the participants suggested that,

“... if government could help with including a boiled egg, a slice of bread and a glass of milk".

Participant 2, FGD 1 
This sort of adjustment would require radical financial support which was seen as deficient by the participants regardless of the value it could bring in bridging the gap caused by skipping breakfast. This was supported by another participant:

"menu needs to be improved and budget be increased".

Participant 3, FGD 2

The NSNP listed the general rules on its implementation and highlighted the desired menu which promoted the use of locally preferred foods. It does not stipulate a strict rule that no variations or substitutions on any given day are permitted.

Participants expressed their desire for the proper implementation of the NSNP but felt that the implementation of the policy became difficult because of the budget provided for it. One participant indicated barriers to the implementation of the NSNP as,

"it will be difficult for the proper implementation with small budget. The vision is high but budget is low".

Participant 2, FGD 2

Participants expressed concerns in the budgetary constraints which were exposing their children to different forms of risks and compromise. One participant suggested that the budgetary barriers were considered as exposing the schools to a compromised quality of foods and said,

"budget prevents proper implementation of the NSNP and we end up buying cheaper foods thus compromising quality and often look for specials which often have close to expiring foods".

Participant 4, FGD 1

This was also echoed in the other FGD where participants expressed the view that the situation was exacerbated by the poor food preparation skills among food handlers.

\subsubsection{Subtheme 4: Food handling and preparations}

Participants felt that on top of the compromised quality of food they bought, food was also not prepared in a palatable manner due to lack of skills by those who prepared it.

"Training of food handlers is needed as preparing meal for a thousand children requires a skill and without a skill food won't be tasty and children won't eat it leading to high food wastages".

Participant 5, FGD 2

\subsection{THEME 2: VENDOR SYSTEM}

\subsubsection{Subtheme 1: Sold items}

Based on the quality of food prepared at school and its palatability, participants indicated that children find themselves having to buy something from the vendors who often do not sell the prescribed items in accordance with the guide for tuck-shops. Participants reported that they had attempted to guide the vendors on what needed to be sold but compliance was very low as they reported that children liked the stuff that was sold to them. One participant expressed that,

"We are aware of dangers of chips and we have started to discuss with vendors to stop the selling of these including sweets. We have asked them to sell healthy staff but we have trouble with compliance".

Participant 5, FGD 2 
The school children eat sweets, fat cakes (fried in oil), chips and dozens of unhealthy items that are prohibited in terms of the NSNP guide. It was reported that compliance by the vendors was challenged by 'soft rules' on what was acceptable inside school premises and what was not. Schools could not enforce anything without a written school policy and support of the district office. Failure to ensure compliance was in one case put to the test: learners who were found with prohibited food items inside school premises were requested to bring a parent to school the following day; however, the parents ignored this. There was also the concept of informing parents that performance reports were going to be withheld until they came to the school but that was also not supported and was opposed by the district office. The participants felt that the district office was not providing adequate support to the prevention of the risk factors and their children were at risk in turn. One participant reported,

"we would have a problem with the district that would call and say release that report to the parent immediately".

Participant 7, FGD 2

It appeared to be common that schools were not at liberty to develop and apply rules in line with the situations they found themselves in. One participant felt that they were not permitted by the district office to be innovative and to apply their minds. As such, they were always at odds with the district office and ended up letting things go just for the sake of peace.

\section{Discussion}

This study assessed the perceptions of school management teams on the association between school nutrition programmes and development of non-communicable diseases among learners. Participants in the study felt that the national school nutrition programme contributed immensely towards changing the situation in their communities and schools as they had needy children. This is supported by Bartfeld and Ahn [18] who reported that the provision of school nutrition programmes contributed meaningfully in situations of food insecurity, leading to children worrying less about food and focusing on educational outcomes.

Healthy nutrition provided by NSNPs has been commended for ensuring that children were kept at school with significantly lowered rates of absenteeism [19]. Improved school attendance together with improved performance were considered a perfect recipe for ensuring improved literacy levels in a society thus leading to improved employment opportunities for the near future. That study established that poverty levels and food insecurity were key factors that contributed to high rates of school dropout and poor academic performance even among those who attended school. It was established that food insecurity led to several irreversible educational and developmental outcomes [20,21] state that the implications of food insecurity could go as far as affecting the behavioural and emotional being of children. To address the food insecurity gap, the US Department of Agriculture [22] expressed support for the establishment of a healthy nutrition environment in school. The US Department of Agriculture acknowledged that there were many factors that played a role in the development of childhood obesity and, as such, school nutrition programmes could play a role in combating childhood obesity.

A suggestion that arises from this present study is that the South African NSNP needs reengineering to include breakfast. The importance of breakfast has been reported as significant to eliminate situations of children who were inactive in classes, children who collapsed in classes because of hunger, and children who had poor academic performance. This suggestion of reengineering of the NSNP to include breakfast was supported by a study conducted in South Africa in 2015 [23]. Addition of breakfast in the school nutrition programme would reduce the effects of skipping breakfast, which is a risk factor for non-communicable diseases like diabetes. There is evidence suggestive that consumption of breakfast contributes to a reduced Body Mass Index and ultimately reduces chances of 
overweight among children and adolescents [24]. These findings were supported by Kesztyüs, et al. [25] who reported that there was association between skipping breakfast with development of abdominal obesity among children in lower grades at school. Kesztyus and colleagues suggested that schools need to offer breakfast before the school day begins on a regular basis. In a study conducted in South Africa in 2012, it was established that there was a strong perception that improved concentration and classroom interactions were strongly associated with breakfast programmes in a studied population [26].

Introduction of breakfast would mean that the design of the school nutrition program should be restructured. This study found that the menus at schools were reported as not being helpful to the school management team in addressing nutritional needs and academic impact. According to the participants, this was due to the imposed and inflexible approach of the district education department through nutrition coordinators who did not accommodate local situations. That imposed approach to the menu did not take into account locally desired and used foods.

The menu in the NSNP according to DBE [7] reflected,

"School menus should offer tasty and adequate meals which must fulfil at least $30 \%$ of the daily nutritional needs of learners per meal. It is important to serve a balanced meal which is composed of:

- Protein:

- $\quad$ vegetable protein e.g. Soya products, dried beans, lentils, nuts and dried peas or

- animal protein e.g. meat, milk, eggs and fish (depending on affordability)

- Starch: e.g. maize meal, samp, mealie rice, rice, bread, potatoes

- Vegetables: at least one green and one red or yellow or orange vegetable per meal"[7].

In line with the provisions of the NSNP, the school menu as prescribed by the district specified items that needed to be adhered to. Participants expressed that flexibility was not provided by the district in the local implementation of the policy or menu. In this sense the menu as provided by the district was not properly consistent with the provisions of the NSNP.

The NSPN further recorded,

"Very important

- $\quad$ Selected menus should be socially acceptable.

- Use of indigenous food in menus is encouraged.

- Specifications of new menu option inclusions are obtainable from the District/Circuit NSNP officer.

Peanut butter may only be used if quality assurance standards by the Department of Health have been met" [7].

Skills development was found necessary to ensure that food was not wasted given minimal resources in schools. The NSNP requires the food handlers to be trained on health and hygiene standards. This would enhance the quality of meals prepared [7]. However, it was reported that the food handlers in the NSNP are often overworked; some are destitute owing to the fact that they are hired on short rotating contracts; and this is exacerbated by unemployability as a result of lack of skills [27]. This often results in poor quality of food being prepared by handlers, which was reported to be a factor leading learners to turn to the vendors to buy fat cakes, chips and sweets and other foods that are NCD risk factors. These undesirable low-cost items sold by vendors pose risks of development of NCD risk factors like obesity. Open availability of the unhealthy foods from vendors encourages children to buy them while reducing their desire to consume healthy food prepared and provided by the school [28]. It transpired in the study that the vendor space needed to be controlled. However, participants felt that more support from the district office was required for the successful control of the space. This support by authorities was reported as only minimally available within nutrition education [29]. 
Reeve and Gostin suggest that the burden of NCDs can be attributed to aggressive marketing of unhealthy food, alcohol and tobacco in LMICs. This includes the major campaigns used by manufacturers that undermine the existence of regulations and guidelines established to reduce behavioral risk factors for NCDs [4]. Such policies, regulations and guidelines are essential in the controlling of what is sold, and in empowering the population on essential information required before one purchases any food product. It has been reported that whenever government administration establish firmer controls to mediate progression of NCDs, the food and beverage industries tend to obstruct public policy development to maximize their gains $[30,31]$.

In an effort to address such interferences by manufacturers, administrations have developed instruments to guide practice of good governance with effective systems to neutralize any potential conflicting interests [32]. Regulatory frameworks are designed to control supply and selling of foods to children, especially during an era in which the demand for and supply of meat, dairy products, sugary beverages, and processed and ultra-processed foods has increased exponentially [33]. With this awareness, the government of South Africa developed a National School Nutrition Programme (NSNP) to address and mediate nutritional disparities within populations [7]. This guiding policy also regulates the establishment of outlets and vendor systems specifying what needs to be sold to children at schools. Branca, Lartey, Oenema, Aguayo, Stordalen, Richardson, Arvelo and Afshin [5] suggest that school nutrition programmes can ensure school children have access to diversified nutrition, important for healthy growth and development. Branca et al. stress that the nutrition programmes could play an important role in limiting children's exposure to unhealthy foods such as are sold in outlets and by vendors.

\section{Limitations}

Even though efforts were made for delimitations, some limitations were faced. The study was limited only to schools where children of the prospective urban rural epidemiology study (PURE) families went and not the overall population of Mt Frere. Parents from these families were eligible of being part of the school governing body and ultimately school management team. As such, it cannot be considered representative of Mt Frere. Nevertheless, the findings of the study illustrate an acceptable understanding of the perceptions on the association between school nutrition programmes and development of future lifestyle diseases among learners. The study did not include all stakeholders necessary for policy development and change, however, this can be best implemented by future studies. Due to limited scope of data, the current study did not adequately determine advantages and disadvantages of the school nutrition program. The sample size of the study could not provide health and nutrition framework themes. Despite all the limitations, this study determined the role of the school nutrition program in fighting poverty among the rural poor children. This resulted in improved school attendance, reduction in drop-out rates and improved throughput. This study remained with value in creating body of knowledge especially on school nutrition programmes in rural communities of South Africa.

\section{Conclusions}

Health Promotion is described by WHO as a process whose results cannot be seen immediately. Enabling people to take control of health determinants would necessitate the development of enabling factors such as policy in this situation and commitment from affected individuals. To improve the NSNP's effectiveness and viability, school authorities must commit to reengineering it at the local level. This process would then take a long time to implement because numerous stakeholders and policy actors would need to be thoroughly consulted. However, the centralization of authority at district office level seems to hinder rather than enhance performance at school level. This suggests that the fight against those NCDs risk factors is going to be a long one. Radical decisions need to be made on how to control vendor space and ensure compliance in the implementation of the guide to vendors. Future studies should consider inclusive implementation research studies where stakeholders necessary to change the policy in school programs or vendor systems are included. Such stakeholders could include school governing bodies, sub-district, district, provincial education and health departments. A study involving the broader 
population is needed for the development of a nutrition and health promotion framework to mitigate the extent of the development of lifestyle diseases.

Author Contributions: SCN: conceptualization, design, data collection, execution and drafting and revision of the manuscript. TRA: funding, supervision and review of the manuscript. SAM: analyses, funding review of the manuscript.

Funding: The work reported herein was made possible through funding by the South African Medical Research Council through its Division of Research Capacity Development under the SAMRC Research Capacity Development Initiative (MRC-RFACC 01-2014), from funding received from the South African National Treasury. The content hereof is the sole responsibility of the authors and do not necessarily represent the official views of the SAMRC or the funders.

Institutional Review Board Statement: Walter Sisulu University's Human Research Ethics and Biosafety Committee for ethical approval (070/15) in accordance with the guidelines of the Declaration of Helsinki.

Informed Consent Statement: Participants were provided with detailed information sheet with full details about the study and reporting channels and they signed a written informed consent.

Data Availability Statement: Data are available on request though it will be guided by research regulations, POPIA and the confidentiality agreement with participants.

Conflicts of Interest: There was no conflicting interest from all authors.

\section{References}

1. WHO, W.H.O. Noncommunicable diseases country profiles 2018. 2018.

2. Chakma, J.K.; Gupta, S. Lifestyle and non-communicable diseases: a double edged sword for future India. Indian Journal of Community Health 2014, 26, 325-332.

3. WHO, W.H.O. Non-communicable Diseases (NCD) Country Profile, 2014. Geneva 2014, 27, 177.

4. Reeve, B.; Gostin, L.O. "Big” Food, Tobacco, and Alcohol: Reducing Industry Influence on Noncommunicable Disease Prevention Laws and Policies. 2019.

5. Branca, F.; Lartey, A.; Oenema, S.; Aguayo, V.; Stordalen, G.A.; Richardson, R.; Arvelo, M.; Afshin, A. Transforming the food system to fight non-communicable diseases. Bmj 2019, 364.

6. Hunter-Adams, J.; Battersby, J. Health care providers' perspectives of diet-related non-communicable disease in South Africa. BMC public health 2020, 20, 1-10.

7. DBE, D.o.B.E. National School Nutrition Programme: A guide for Secondary School. 2009.

8. Gresse, A.; Nomvete, A.; Walter, C. Situational analysis: Implementation of the National School Nutrition Programme in low socio-economic primary schools in Nelson Mandela Bay. Journal of Consumer Sciences 2017.

9. Bush, T.; Glover, D. School management teams in South Africa: A survey of school leaders in the Mpumalanga Province. International Studies in Educational Administration (Commonwealth Council for Educational Administration \& Management (CCEAM)) 2013, 41.

10. Kroone, D.; Alant, B. Competing with the tuck shop: How can I influence teenage food choices in this South African high school? Educational Research for Social Change (ERSC) 2012, 1, 69-84.

11. Donald, D.; Dawes, A.; Louw, J. Addressing childhood adversity; New Africa Books: 2000.

12. Skinner, D. Multiple vulnerabilities: Qualitative data for the study of orphans and vulnerable children in South Africa; HSRC Press: 2006.

13. Shisana, O.; Labadarios, D.; Rehle, T.; Simbayi, L.; Zuma, K.; Dhansay, A.; Reddy, P.; Parker, W.; Hoosain, E.; Naidoo, P. The South African National Health and Nutrition Examination Survey, 2012: SANHANES-1: the health and nutritional status of the nation. 2014.

14. Shrestha, R.M.; Ghimire, M.; Shakya, P.; Ayer, R.; Dhital, R.; Jimba, M. School health and nutrition program implementation, impact, and challenges in schools of Nepal: stakeholders' perceptions. Tropical Medicine and Health 2019, 47, 32, doi:10.1186/s41182-019-0159-4.

15. Statistics South Africa, S.S.A. Community Survey 2016; Statistics South Africa: Pretoria, 20182018.

16. Creswell, J.W.; Creswell, J.D. Research design: Qualitative, quantitative, and mixed methods approaches; Sage publications: 2017.

17. Lincoln, Y.S.; Lynham, S.A.; Guba, E.G. Paradigmatic controversies, contradictions, and emerging confluences, revisited. The Sage handbook of qualitative research 2011, 4, 97-128. 
18. Bartfeld, J.S.; Ahn, H.-M. The School Breakfast Program strengthens household food security among low-income households with elementary school children. The Journal of nutrition 2011, 141, 470-475.

19. Jamaluddine, Z.; Choufani, J.; Masterson, A.R.; Hoteit, R.; Sahyoun, N.R.; Ghattas, H. A Community-Based School Nutrition Intervention Improves Diet Diversity and School Attendance in Palestinian Refugee Schoolchildren in Lebanon. Current Developments in Nutrition 2020, 4, doi:10.1093/cdn/nzaa164.

20. Jamaluddine, Z.; Sahyoun, N.R.; Choufani, J.; Sassine, A.J.; Ghattas, H. Child-reported food insecurity is negatively associated with household food security, socioeconomic status, diet diversity, and school performance among children attending UN Relief and Works Agency for Palestine Refugees schools in Lebanon. The Journal of nutrition 2019, 149, 2228-2235.

21. Shankar, P.; Chung, R.; Frank, D.A. Association of food insecurity with children's behavioral, emotional, and academic outcomes: a systematic review. Journal of Developmental \& Behavioral Pediatrics 2017, 38, 135-150.

22. US Department of Agriculture, U. National School Lunch Program and School Breakfast Program: nutrition standards for all foods sold in school as required by the Healthy, Hunger-Free Kids Act of 2010-interim final rule. Fed Regist 2013, 78, 3906839120.

23. Graham, L.; Hochfeld, T.; Stuart, L. Double trouble: addressing stunting and obesity via school nutrition. South African journal of child health $2018,12,90-94$.

24. de la Hunty, A.; Gibson, S.; Ashwell, M. Does regular breakfast cereal consumption help children and adolescents stay slimmer? A systematic review and meta-analysis. Obesity facts 2013, 6, 70-85.

25. Kesztyüs, D.; Traub, M.; Lauer, R.; Kesztyüs, T.; Steinacker, J.M. Skipping breakfast is detrimental for primary school children: cross-sectional analysis of determinants for targeted prevention. BMC public health 2017, 17, 258, doi:10.1186/s12889-017-4169$\mathrm{z}$.

26. Hochfeld, T.; Graham, L.; Patel, L.; Moodley, J.; Ross, E. Does school breakfast make a difference? An evaluation of an in-school breakfast programme in South Africa. International Journal of Educational Development 2016, 51, 1-9.

27. Berejena, T.; Kleynhans, I.; Vibetti, S. Economic impacts of the rotational policy on the food handlers in the national school nutrition programme in gauteng province. International journal of economics and finance 2020, 12.

28. Bauer, K.W.; Yang, Y.W.; Austin, S.B. "How can we stay healthy when you're throwing all of this in front of us?" Findings from focus groups and interviews in middle schools on environmental influences on nutrition and physical activity. Health Education \& Behavior 2004, 31, 34-46.

29. Kupolati, M.D.; Gericke, G.J.; MacIntyre, U.E. Teachers' perceptions of school nutrition education's influence on eating behaviours of learners in the Bronkhorstspruit District. South African Journal of Education 2015, 35, 1049-1049.

30. Martino, F.P.; Miller, P.G.; Coomber, K.; Hancock, L.; Kypri, K. Analysis of alcohol industry submissions against marketing regulation. PloS one 2017, 12, e0170366.

31. Mialon, M.; Swinburn, B.; Wate, J.; Tukana, I.; Sacks, G. Analysis of the corporate political activity of major food industry actors in Fiji. Globalization and health 2016, 12, 18.

32. Tangcharoensathien, V.; Chandrasiri, O.; Kunpeuk, W.; Markchang, K.; Pangkariya, N. Addressing NCDs: challenges from industry market promotion and interferences. International Journal of Health Policy and Management 2019, 8, 256.

33. Scott, P. Global panel on agriculture and food systems for nutrition: food systems and diets: facing the challenges of the 21st century. Food Security: The Science, Sociology and Economics of Food Production and Access to Food 2017, 9, 653-654. 